\title{
Intelligent Computing: Oxymoron? ${ }^{+}$
}

\author{
Marcin J. Schroeder \\ Global Learning Center, Tohoku University, Sendai 980-8576, Japan; schroeder.marcin.e4@tohoku.ac.jp; \\ Tel.: +81-22-795-3246 \\ + Conference Morphological, Natural, Analog and Other Unconventional Forms of Computing for Cognition \\ and Intelligence, Berkeley, CA, USA, 2-6 June 2019. \\ Published: 7 May 2020
}

\begin{abstract}
The present paper is intended as a defense of the view that whatever makes AI so useful and successful in competition with humans, it does not have to be intelligence, at least as we use this term in the human context. After all, the question of qualification for human beings to be considered intelligent does not have a definite answer. There is not even a common agreement on whether we can identify criteria for one (general or unified) intelligence or whether we should rather consider multiple intelligences. Thus, our discussion is focusing not on a complete definition of intelligence, but only on its necessary conditions, and not on computing artefacts, but on the process of computing in its Turing Machine model. Are there any features of computing which are in contradiction with the idea of intelligence? This is the reason why the title of this paper is formulated in a negative way, with the question of whether intelligent computing is an oxymoron.
\end{abstract}

Keywords: artificial intelligence; intelligence; multiple intelligences; information integration; complexity; reduction of complexity

\section{Introduction}

The 2019 Summit of International Society for the Study of Information, held 2-6 June 2019 at The University of California at Berkeley, addressed a large variety of topics related to information and its multiple roles in the fundamental intellectual and technological controversies of the present time. It is not a surprise that the title of the summit, which was formulated as a question "Where is the I in AI and the meaning of Information?" referred to artificial intelligence. However, the question about I in AI may be surprising. Do we have to study, inquire, and analyze intelligence of AI, which wins a competition against human champions of chess or go, the games which for majority of people symbolize high cognitive achievement? What, if not intelligence of AI, pushes many occupations in which automated systems can be not only cheaper, but also better, into oblivion?

The present paper is intended as a defense of the view that whatever makes AI so useful and successful in competition with humans, it does not have to be intelligence, at least as we use this term in the human context. After all, the question of qualification for human beings to be considered intelligent does not have a definite answer. There is not even a common agreement on whether we can identify criteria for one (general or unified) intelligence or whether we should rather consider multiple intelligences. Moreover, human intelligence and its components are subject to assessment on a scale of a wide range. We do not ask what human intelligence can do, but how intelligent is the behavior or actions of a particular human being. The common expression about AI, frequent not only in mass media, but also in philosophical works on the subject, is that it can do this or that, which shows how deeply the concept of AI is mystified. Instead of considering artefacts equipped with sets of functions allowing them to perform complex actions, there is a reference to a mysterious and elusive entity AI which can "do" a lot, and one day may escape our control or even enslave us. This is not just a convenient, harmless abbreviation, when we recognize that the similar use of the 
abbreviation HI, for the discussion of the characteristics of diverse human beings, would have impoverished the study of intelligence in human context.

There are of course some functions or actions of artefacts equipped with mechanisms involving the standard, Turing Machine model of computing which every such machine can perform, and some which none can. For instance, no artificial system equipped exclusively with a computer (i.e., Turing Machine) can produce a sequence of random numbers in the strict sense of the term. This is not an expression of belief or empirical statement, but a simple consequence of the definition of random numbers, i.e., numbers which cannot be produced by an algorithmic process shorter than the resulting sequence of numbers. This does not generate heated discussions, because humans are even worse in generating random numbers. This latter well known feature of humans can be easily accepted as a result of biological evolution, which very likely promoted the ability to detect patterns in the environment (especially those that were dangerous) with the relatively low cost of detection of those that were non-existent. A person who tries to create a sequence of random numbers will always try to avoid sequences which seem to follow patterns, and the result becomes highly non-random.

The example of the impossibility to generate random numbers, although not relevant for the study of intelligence, shows the direction of our study of intelligent computing. Are there any features of computing (not of artefacts, but of the process itself) which are in contradiction with the idea of intelligence? This is the reason why the title of this paper is formulated in a negative way, with the question of whether intelligent computing is an oxymoron. Of course, this methodology cannot be used in the positive way. From the fact that there is no contradiction between the definition of computing and the idea of intelligence, we cannot conclude that computing is intelligent or that intelligence is computational. However, even in the absence of the complete definition of intelligence, but with some of its necessary conditions, if we find them to be in contradiction with the model of computing, we can claim that calling this type of computing intelligent is an oxymoron.

\section{Intelligence}

The systematic study of intelligence of human beings and animals started in the 19th century and continued through the entire 20th century without ever reaching the point where everyone was satisfied with the outcomes. The confounding factor was the normative character of the term "intelligent" in the common sense discourse, which generated many emotional reactions within the academic community and in the general public. The decision to select some traits as qualifying for higher intelligence (and therefore qualifying as a "better" individual) can be used (and actually was sometimes used) as a tool for discrimination within human collectives or between human collectives. The need for the separation of several modes of intelligence and preventing oversimplified comparisons of the better-worse type was already recognized by the pioneers of the discipline, such as Alfred Binet at the turn of the 19th and 20th centuries. Later, the range of components of intelligence was expanded to as many as 120 separate abilities, organized into at least three dimensions (e.g., dimensions of character abilities consisting of contents, operations, and products). It is not a surprise that the concept of intelligence is so complex, as it is not a surprise that there was such big effort in finding some prioritization of the abilities which are of special importance for the assessment of higher intelligence.

It would have been pointless to review hundreds of competing definitions of human intelligence based on a selection of particular desired characteristics of higher priority. As an example, one of many similar claims in the literature on the subject is that intelligence is an ability to understand complex ideas, to adapt to the environment, to learn from experience, and to engage in reasoning to overcome obstacles [1]. Typically, everyone would agree that all abilities included in this definition characterize intelligence, but the issue of priorities and excluded abilities leads to unavoidable disagreements. Thus, the question of conditions qualifying an object, whether artificial or natural, as an entity, or its functioning as intelligent, is still more about the qualifier (intelligent) than the qualified. Of course, if we had an established definition of intelligence, then we could sort 
objects, actions, and processes accordingly. However, we don't. We don't have a clear separation of the qualifications for being intelligent from being creative, talented, innovative, a successful leader, etc.

Turing attempted to escape the problem in the context of artificial systems ("machines") using his "Imitation Game", at present called a "Turing Test" [2], but instead of closing the discussion of "intelligent machinery", he opened a Pandora's box of ever-lasting disputes in which machine intelligence is frequently mixed with the ability to think, a capacity of being conscious, etc. It does not mean that the problem is restricted to intelligent artefacts and clearly separated from the difficulties with human intelligence. So, whether computers or machines can be intelligent or not depends on the way we understand intelligence, and there is no reason to claim that this understanding can be achieved without the distinction of multiple intelligences or multiple components of intelligence.

Of course, this freedom of the choice of their definitions has some limits coming from an already established tradition of the use of the term "intelligence", in particular in the common sense discourse. From this perspective, we cannot ignore the most frequent objection to the intelligence of artefacts based on the doubt that they can have the capacity of symbolic association between a sign and its denotation. Thus, they do not have the capacity to "understand" the meaning of symbols. The objection is not surprising, and its source is not new. One of most discussed objections to Turing's Imitation Game was the "Chinese Room" thought experiment proposed by John Searle in the 1980's. This thought experiment was intended as a demonstration that the imitation of intelligent behavior may hide an actual total lack of understanding without which it is difficult to assess intelligence [3]. We can trace the issue much earlier to Brentano's claim that intention (associating denotation with signs) is a specific mental capacity of the mind which is absent on the body side of the mind-body division.

The criticism of the possibility of intelligent artefacts (or in the more remote past of "thinking body" as opposed to "thinking mind") is typically based on philosophical arguments referring to the fundamental ontological positions, which are the matter of choice, not of discussion. Thus, it is better to rephrase the question about intelligent artefacts to be more suitable for the reflection on computing, not on the mind-body problem in its present formulation in terms of artificial embodiment of cognition.

\section{Intelligent Computing}

Everyone agrees that computing based on the model of the Turing Machine is a transformation of compound symbols (sequences built of digits, Tukey's bits 0 and 1, letters, or any other finite number of elementary units from which symbols are built) through the manipulation of their components. The individual components (digits) have their meaning for the machine, which is determined by instructions and which is expressed in the machine's actions. It was originally stated by Turing that the machine (we would say "head") can see or scan the present square (unitary component of the "tape") for the unitary sign-digit and can act according to the present instruction (state of its "mind"). The action of the machine or, more exactly, its head, can be understood as an expression of the meaning of the unitary sign for the machine [4].

Obviously, a machine's head does not have any representation of the tape with its configuration of digits, nor even any sensor to monitor more than one square (or, in some variations of Turing machines, a fixed, finite number of squares). Thus, a machine cannot "understand" or even "perceive" the entire compound symbol consisting of a possibly long configuration of digits, no matter how we define understanding. Someone could object that the tape is a part of the machine, so machine has a representation of the configuration on the tape in the form of the tape. This, however, kills the concept of the symbolic representation by restricting symbolic representation to the strict identity. Human beings are definitely capable of symbolic representation beyond the meaning understood as the identity of the sign and denotation.

Does this mean that the Turing Machine type computers are doomed to be non-intelligent? No. Someone can claim that the meaning is emergent in both human symbolic thinking and in computing. The difference between actual typical implementations of computers using Tukey's bits 
(0 and 1) and typical human brains with a possibly large, but finite set of elementary units may be misleading. Nothing prevents us from using an implementation of the Turing machine with a finite, but very large set of digits. The view that the meaning is emergent and eliminates the distinction between semantics and syntactic may seem exotic, but not without precedence. After all, it is at the foundation of constructivism. There is nothing fallacious in the assumption that we live in a reality which our mind constructs from a finite number of elementary units ("digits"). Maybe, we actually understand only the finite number of simple components, and we react to the instructions telling our brain how to construct a complex and diverse reality. However, if this is the case, how do we know that the reality is complex and diverse, if we can understand only simple components? How can we direct our actions to eliminate complexity and diversity? In the case of computers (or Turing machines) we know that a Turing machine cannot compute the shortest program producing the same outcome (Kolmogorov-Chaitin algorithmic complexity measure [5,6]) or reduce the algorithmic complexity of the configuration on the tape. It cannot even assess the computability of the input configuration.

Computing is a one-way process of construction, but not deconstruction. It is a human programmer who decomposes in the process of programming the complex task into an algorithm (intelligent part of the task) and leaves the non-intelligent task of performing the construction of the desired outcome. This is not far from the objection to the intelligence of computers coming from the common sense discussions. When we compare the intelligence of different people, we consider as more intelligent the individuals who have the ability to reduce complexity, usually by making complex tasks simple through deconstruction, and leaving these simple tasks to less intelligent collaborators. The example of a definition of intelligence in the previous section refers to complexity too. Can we expect that intelligence can be conceptualized without the ability to reduce complexity, for instance, complexity of information? Thus, the answer given by the author in this part of the paper is: Yes, oxymoron. Computing cannot reduce complexity, and therefore is lacking one of the critical characteristics of intelligence.

\section{Information Integration}

The question remains about what type of capacities have to be added to a Turing machine to make it intelligent at a level comparable with human intelligence or beyond. A partial answer to this question was given by the author in his earlier publications in the context of consciousness $[7,8]$. Computers have to be equipped with the ability to integrate information.

The issue of the integration of information is highly non-trivial. Shannon's mathematical model of communication suffered similar deficiency in performing information integration, which forced him to exclude the meaning of information from consideration [9]. His measure of information, entropy, depends only on probabilities of the components in the message (letters), but not on compound configurations of letters, which are carriers of the meaning. Neither the formula for entropy, nor the diagrammatic description of communication of information, involves a mechanism for information integration. For the same reason, a Turing Machine as a model of computing excludes even a theoretical possibility to process meaning which is necessarily carried by compound symbols. Without compound symbols, computing is impossible. For the Turing Machine, there are distinguishable units (cells or squares on the tape) or the entire tape, nothing in between.

In order to resolve the problem of the meaning of compound symbols presented above, we cannot and do not claim that computers have to be conscious. It is just a human (possibly animal) way to integrate information through presenting the result of integration as a phenomenal conscious experience. The lack of any information integrating mechanism in a Turing Machine not only demonstrates the difference between cognitive processes in a human being and in a computer, but excludes the possibility of any alternative mechanism justifying the qualification for intelligence.

Finally, without the ability to analyze integrated portions of information on the tape, a Turing Machine lacks the ability to use abstraction as a tool for the reduction of complexity. Once again, the obstacle in performing abstract analytic processes is the absence of any mechanism which can recognize which subsequences of unitary signs (digits) are meaningful. A Turing Machine can 
perform operations at the lowest level of abstraction which, due to the high speed of actual realization of a computer, may be sufficient for practical applications. However, it cannot create an additional, lower level of abstraction, which serves as a set theoretical model for the concepts arising at the original level, as meaningful configurations of digits. At least, this cannot be achieved by the machine without the engagement of a human mediator.

Funding: This research received no external funding.

Conflicts of Interest: The author declares no conflict of interest.

\section{References}

1. Neisser, U. Intelligence: Knowns and unknowns. Am. Psychol. 1996, 51, 77-101.

2. Turing, A.M. Computing Machinery and Intelligence. Mind 1950, 59, 433-60.

3. Searle, J. Minds, Brains, and Programs. Behav. Brain Sci. 1980, 3, 417-424.

4. Turing, A.M. On computable numbers, with an application to the Entscheidungsproblem. Proc. Lond. Math. Soc. Ser. 2 1936, 58, 345-363.

5. Kolmogorov, A.N. Three approaches to the definition of the "quantity of information". Probl. Peredachi Informatsii 1965, 1, 3-11.

6. Chaitin, G.J. On the Length of Programs for Computing Finite Binary Sequences. JACM 1966, 13, 547-569.

7. Schroeder, M.J. Quantum Coherence without Quantum Mechanics in Modeling the Unity of Consciousness. In International Symposium on Quantum Interaction; Bruza, P.; Ed.; Springer: Heidelberg, Germany, 2009; Volume 5494, pp. 97-112.

8. Schroeder, M.J. Concept of Information as a Bridge between Mind and Brain. Information 2011, 2, 478-509.

9. Shannon, E.C.; Weaver, W. The Mathematical Theory of Communication; University of Illinois Press: Urbana, IL, USA, 1949.

(C) 2020 by the authors. Licensee MDPI, Basel, Switzerland. This article is an open access article distributed under the terms and conditions of the Creative Commons Attribution (CC BY) license (http://creativecommons.org/licenses/by/4.0/). 